TITLE:

\title{
Criterion for decrease of electric field at ionization point of liquid- metal ion sources
}

$\operatorname{AUTHOR}(\mathrm{S}):$

Gotoh, Y; Tsuji, H; Ishikawa, J

\section{CITATION:}

Gotoh, Y ... [et al]. Criterion for decrease of electric field at ionization point of liquid-metal ion sources. REVIEW OF SCIENTIFIC INSTRUMENTS 2000, 71(2): 725-727

\section{ISSUE DATE:}

2000-02

URL:

http://hdl.handle.net/2433/39797

\section{RIGHT:}

Copyright 2000 American Institute of Physics. This article may be downloaded for personal use only. Any other use requires prior permission of the author and the American Institute of Physics. 


\title{
Criterion for decrease of electric field at ionization point of liquid-metal ion sources
}

\author{
Y. Gotoh, ${ }^{\text {a) }}$ H. Tsuji, and J. Ishikawa \\ Department of Electronic Science and Engineering, Kyoto University, Yoshida-honmachi, Sakyo-ku, \\ Kyoto 606-8501, Japan
}

(Presented on 6 September 1999)

Criterion for decrease of electric field at the ionization point of liquid-metal ion sources has been derived. The argument is based on the equation of space-charge limited current with small charge approximation and the balance of electric field stress, with liquid flow and surface tension stress. The result was such that it is possible to decrease the electric field with an increase in the applied voltage, that is, the source current, under the condition of weaker dependence of current density on the electric field than that in the conventional low temperature field evaporation theory. The dependence of the ion current density on the electric field was calculated from the dependence of the angular current intensity on the electric field, assuming postionization theory. The result indicated that the dependence became almost proportional, which is surprisingly weak for field ion emission.

(C) 2000 American Institute of Physics. [S0034-6748(00)57002-6]

\section{INTRODUCTION}

It has been believed that the ionization mechanism of liquid-metal ion sources (LMISs) is field evaporation, ${ }^{1}$ but it is still difficult to say that the theoretical establishment has been made. Both image hump model ${ }^{2}$ and charge exchange model $^{3,4}$ failed to show the experimentally obtained charge state distribution $\left(R_{21}\right.$, atomic intensity of doubly charged ions to singly charged ions). For the charge state distribution, postionization model ${ }^{5}$ is known to be superior, this model requires evaporation model prior to the postionization. Thus, the mechanism of liquid-metal ion sources is left unknown.

We have been investigating the mass spectra of some element ion sources ${ }^{6}$ and alloy ion sources. ${ }^{7}$ As a result, the $R_{21}$ decreased with an increase in the source current at the regime higher than $10 \mu \mathrm{A}$. The reason of this decrease in $R_{21}$ would be primarily attributed to the decrease of electric field at the ionization point. Generally, it is difficult to accept that the electric field decreases with an increase in the applied voltage. Here in this article, we discuss the criterion for this decrease of electric field with an increase in the source current.

\section{DEPENDENCE OF ELECTRIC FIELD ON SOURCE CURRENT BASED ON POSTIONIZATION THEORY}

Here we first derive the relation between the electric field and the source current from the experimentally obtained $R_{21} \cdot R_{21}$ was measured by a sector magnet. Details in the measurements are described in Ref. 6. We can obtain charge state distribution calculated by postionization theory from Ref. 5 . We have fitted the curve by

$$
\ln R_{21}=A+B \ln \sqrt{F},
$$

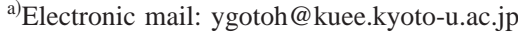

where $A$ and $B$ are fitting parameters. Once $A$ and $B$ are determined, we can easily derive the electric field at the ionization point from the above equation. The values of $A$ and $B$ are listed in Table I. Figure 1 shows the relation between the electric field $F$ and the angular current intensity $I_{\Omega} . I_{\Omega}$ was defined by the intensity of singly charged ions against the source current. We have assumed that the electric field can be expressed as

$$
\ln F=C+D_{\Omega} \ln I_{\Omega},
$$

where $C$ and $D_{\Omega}$ are fitting parameters. From this equation, we may write

$$
I_{\Omega}=\exp (-C) F^{E},
$$

where $E=1 / D_{\Omega}$. The obtained values are denoted in Table I. From this table, it is noted that the current is inversely proportional to the tenth to the fiftieth power of the electric field.

\section{CRITERION FOR DECREASE OF ELECTRIC FIELD}

\section{A. Space-charge limited current with small charge approximation}

The equation of space charge limited current with small charge approximation ${ }^{8,9}$ holds where the electric field at the ionization point is not zero,

$$
F_{L}^{2}-F_{P}^{2}=\frac{c}{\epsilon_{0}} J \sqrt{V},
$$

where $F_{L}$ and $F_{P}$ are Laplace and Poisson field at the apex, $\epsilon_{0}$ is permittivity of vacuum, $c=4 / 3 \sqrt{m / e}, J$ is the current density, and $V$ is the applied voltage between emitter and counter electrode. Generally, the Laplace field $F_{L}$ can be written as

$$
F_{L}=\frac{V}{k r_{a}},
$$


TABLE I. Coefficients of $A, B, C$, and $D$ obtained from the experimental results.

\begin{tabular}{cccccc}
\hline \hline Element & $A$ & $B$ & $C$ & $D_{\Omega}$ & $E$ \\
\hline $\mathrm{Cu}$ & $-4.90 \times 10^{1}$ & 8.81 & 3.46 & $-1.06 \times 10^{-1}$ & -9.43 \\
$\mathrm{Au}$ & $-3.86 \times 10^{1}$ & 6.55 & 3.58 & $-4.46 \times 10^{-2}$ & -22.4 \\
$\mathrm{Sn}$ & $-4.80 \times 10^{1}$ & 11.7 & 2.84 & $-1.85 \times 10^{-2}$ & -54.1 \\
\hline \hline
\end{tabular}

where $k$ is a coefficient weakly dependent on $r_{a}$. If the changes of $F_{P}$ is small, differentiating both sides, we obtain

$$
2\left[\left(\frac{V}{k r_{a}}\right)^{2}-\frac{c}{4 \epsilon_{0}} J \sqrt{V}\right] \frac{d V}{V}=2\left(\frac{V}{k r_{a}}\right)^{2} \frac{d r_{a}}{r_{a}}+\frac{c}{\epsilon_{0}} J \sqrt{V} \frac{d J}{J} .
$$

Taking $\left(V / k r_{a}\right)^{2} \sim\left(c / \epsilon_{0}\right) J \sqrt{V}$ into consideration, Eq. (6) can be reduced to

$$
\frac{3}{2} \frac{d V}{V}=2 \frac{d r_{a}}{r_{a}}+\frac{d J}{J} .
$$

As shown experimentally, the current density, i.e., the electric field $F_{P}$ decreases with an increase in the applied voltage. To allow this, the right-hand side of Eqs. (6) or (7) should be positive, even though the second term is negative. This means that the apex radius $r_{a}$ should increase with an increase in the voltage. The dependence of the current density on the field should satisfy

$$
2 \frac{d r_{a}}{r_{a}}>-\frac{d J}{J}
$$

This can be written as

$$
J \propto \frac{1}{r_{a}^{n}}, \quad n<2 .
$$

This is a general equation of criterion for decrease of electric field with an increase of the voltage. To apply this equation to LMIS, balance at the apex should be included in the present equation.

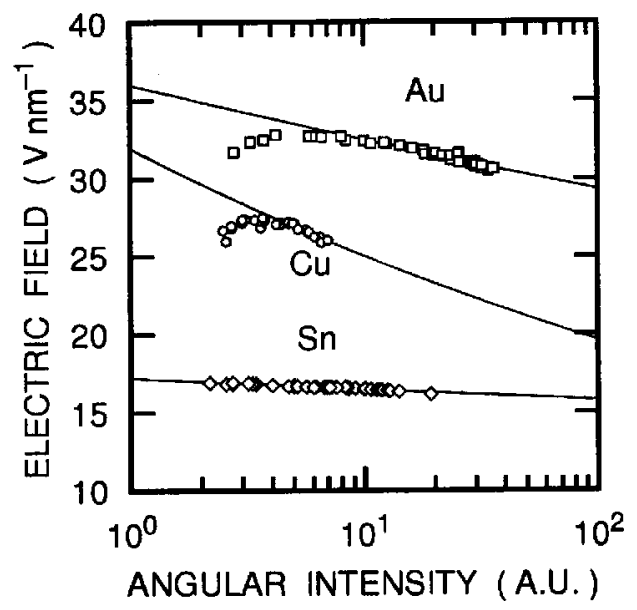

FIG. 1. Relation between electric field $F$ and the angular current intensity $I_{\Omega}$.

\section{B. Required relation between electric field and current density}

The balance at the apex ${ }^{10}$ can be written as

$$
S_{F}=\frac{1}{2} \epsilon_{0} F_{P}^{2}=\frac{1}{2} \rho \nu^{2}+\frac{2 \gamma}{r_{a}}=\frac{1}{2} \rho\left(\frac{J}{e N_{\mathrm{v}}}\right)^{2}+\frac{2 \gamma}{r_{a}}=K+S_{T},
$$

where $\rho$ is the weight density, $N_{\vee}$ is the atomic density, and $\gamma$ is the surface tension of the liquid. $S_{F}, K$, and $S_{T}$ are electric field stress, kinetic energy of liquid, and surface tension stress, respectively. If massive flow does not exist, that is, reverse mode, kinetic energy term disappears. Differentiating both sides of Eq. (10), we obtain

$$
\begin{aligned}
\frac{d J}{J} & =\frac{\frac{1}{2} \epsilon_{0} F_{P}^{2}}{\frac{1}{2} \rho\left(J / e N_{\mathrm{v}}\right)^{2}} \frac{d F_{P}}{F_{P}}+\frac{1}{2} \frac{2 \gamma / r_{a}}{\frac{1}{2} \rho\left(J / e N_{\mathrm{v}}\right)^{2}} \frac{d r_{a}}{r_{a}} \\
& =\frac{S_{F}}{K} \frac{d F_{P}}{F_{P}}+\frac{1}{2} \frac{S_{T}}{K} \frac{d r_{a}}{r_{a}} .
\end{aligned}
$$

Substituting Eq. (11) to Eq. (6),

$$
\frac{3}{2} \frac{d V}{V}=2\left[1+\frac{1}{4} \frac{S_{T}}{K}\right] \frac{d r_{a}}{r_{a}}+\frac{S_{F}}{K} \frac{d F_{P}}{F_{P}} .
$$

From this equation, it is possible that both $r_{a}$ and $F_{P}$ can increase with an increase in $V$, but also it is possible that either of $r_{a}$ or $F_{P}$ increases and the other decreases. Whether the case is the former or the latter is determined by setting the right-hand side is equal to zero, thus

$$
2\left[1+\frac{1}{4} \frac{S_{T}}{K}\right] \frac{d r_{a}}{r_{a}}+\frac{S_{F}}{K} \frac{d F_{P}}{F_{P}}=0 .
$$

From Eq. (13), using $S_{F}=K+S_{K}$,

$$
F_{P} \propto r_{a}^{-2 \frac{1+S_{T} / 4 K}{S_{F} / K}}=r_{a}^{-2 \frac{1+S_{T} / 4 K}{1+S_{T} / K}} .
$$

The magnitudes of $S_{T}, K$, and $S_{F}$ are not known and some researchers assume differently. For example, Kingham and Swanson ${ }^{10}$ treated the equation with no limitation of magnitudes of these terms, which results in $K$ dominating the right hand. Forbes ${ }^{11}$ treated $S_{T}$ in balance with $S_{F}$ and $\mathrm{We}^{6}$ assumed, the liquid cone is terminated where $K \sim S_{T}$. We define these different treatment case I: Kingham and Swanson, case II: Gotoh et al., and case III: Forbes. For each of them, the critical relation is,

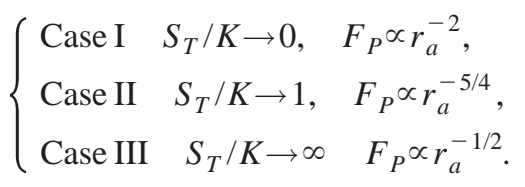

For the current density, substituting Eq. (14) to Eq. (11),

$$
\frac{d J}{J}=\left[1+\frac{3 S_{T} / K}{4+S_{T} / K}\right] \frac{d F_{P}}{F_{P}} .
$$

Thus in a similar way with Eq. (15), critical relation between $J$ and $F_{P}$ is 

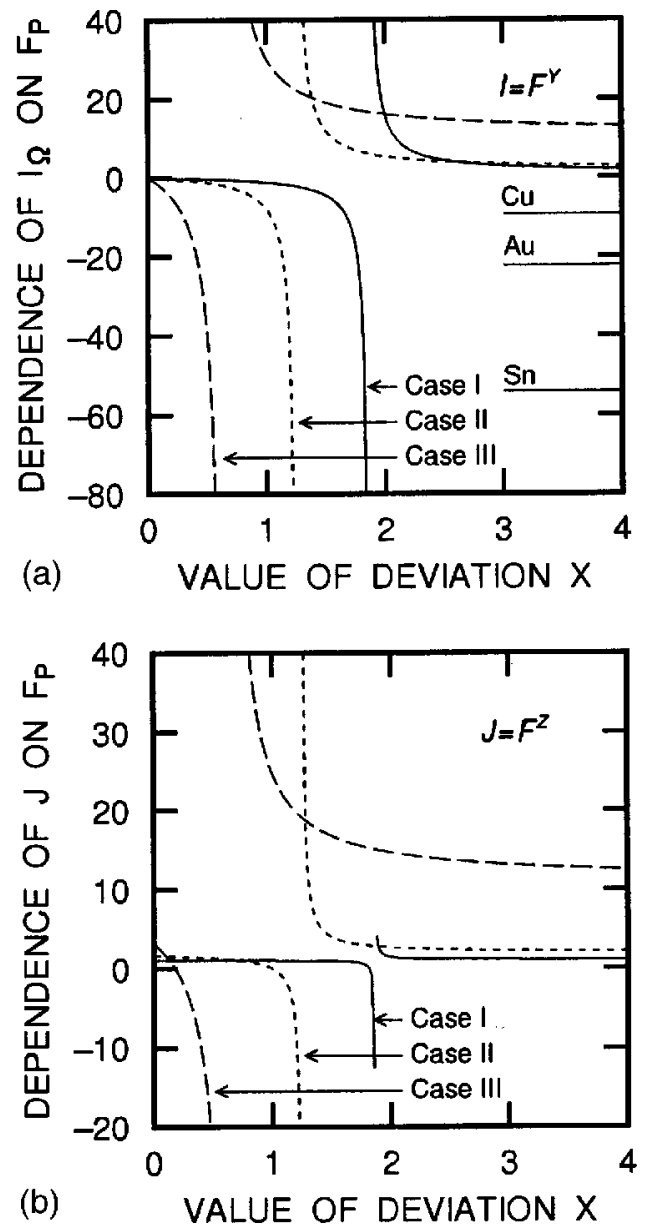

FIG. 2. Dependence of angular current intensity $I_{\Omega}$ and current density $J$ as a function of $X$. (a) Dependence of $I_{\Omega}$ on $X$, and (b) dependence of $J$ on $X$.

$$
\left\{\begin{array}{lcc}
\text { Case I } & S_{T} / K \rightarrow 0, & J \propto F_{P}, \\
\text { Case II } & S_{T} / K \rightarrow 1, & J \propto F_{P}^{8 / 5}, \\
\text { Case III } \quad S_{T} / K \rightarrow \infty & J \propto F_{P}^{4} .
\end{array}\right.
$$

From this equation, to decrease the electric field as the source current increases, i.e., as the external voltage increases, dependence of the current density on the electric field should be very weak.

\section{Dependence of emission current and current density on electric field}

It is necessary to check the validity of the above argument with the experimental results. Experimentally observable quantity is current and not current density. Here considering the angular current $I_{\Omega}$,

$$
\frac{d I_{\Omega}}{I_{\Omega}}=2 \frac{d r_{a}}{r_{a}}+\frac{d J}{J} .
$$

If the relation between apex radius and electric field deviates from the critical equation, we may write,

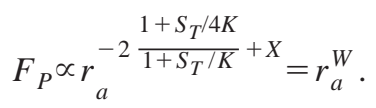

Equation (16) will be

$$
\frac{d J}{J}=\left[1+\left(1+\frac{1}{2 W}\right) \frac{S_{T}}{K}\right] \frac{d F_{P}}{F_{P}}=Z \frac{d F_{P}}{F_{P}} .
$$

This equation is reduced to Eq. (16) when $X=0$. And Eq. (18) will be

$$
\frac{d I_{\Omega}}{I_{\Omega}}=\frac{X\left(1+S_{T} / K\right)}{W} \frac{d F_{P}}{F_{P}}=Y \frac{d F_{p}}{F_{P}} .
$$

Figures 2(a) and 2(b) show the dependence of $I_{\Omega}$ on $F_{P}$ and $J$ on $F_{P}$, that is, $Y$ and $Z$ as a function of $X$. Solid, dashed, and broken lines represent the characteristics for cases I, II, and III, respectively. Here it should be noted $K=0$ gives divergence of the relation. For cases I and III, $S_{T} / K$ was taken as 0.1 and 10. In Fig. 2(a), relation for $\mathrm{Cu}, \mathrm{Au}$, and $\mathrm{Sn}$ ion sources calculated with postionization theory is indicated. Assume case I, if $X<2$, then $d I_{\Omega}>0$ with $d F_{P}<0$, which agrees with the experimental result. However, taking the fact into consideration that dependence of $F_{P}$ on $I_{\Omega}$ is approximately the tenth or fiftieth power, $X$ should be close to 2 . For case III, $X<1 / 2$ is necessary and also $X$ should be close to $1 / 2$. Under such a situation, dependence of $J$ on $F_{P}$ becomes very weak. For $\mathrm{Cu}, E=-9.43=Y$ is realized at $X$ $=1.67$, and for $\mathrm{Au}, E=-22.4=Y$ is realized at $X=1.78$. Obtained index of relation $Z$ 's are 0.84 and 0.5 , respectively. These values are surprisingly small for field evaporation. This may be attributed to the first assumption. Field dependence of postionization theory is still strong, and if dependence of $I_{\Omega}$ on $F$ is weaker, dependence of $J$ on $F$ becomes stronger, and vice versa.

\section{CONCLUSION}

In this study, criterion for decrease of the electric field at the ionization point of liquid-metal ion sources has been derived. The present study revealed that the dependence of current density on the electric field should be lower than the conventional low temperature field evaporation theory. This may give a useful information for the analysis of emission mechanism of liquid-metal ion sources.

${ }^{1}$ L. W. Swanson and D. R. Kingham, Appl. Phys. A 41, 223 (1986).

${ }^{2}$ E. W. Müller, Phys. Rev. 102, 618 (1956).

${ }^{3}$ R. Gomer, J. Chem. Phys. 31, 341 (1959).

${ }^{4}$ R. Gomer and L. W. Swanson, J. Chem. Phys. 38, 1613 (1963).

${ }^{5}$ D. R. Kingham, Surf. Sci. 116, 273 (1982).

${ }^{6}$ Y. Gotoh, T. Kashiwagi, H. Tsuji, and J. Ishikawa, Appl. Phys. A 64, 527 (1997).

${ }^{7}$ Y. Gotoh, H. Tsuji, and J. Ishikawa, Ultramicroscopy 73, 83 (1998).

${ }^{8}$ T. E. Stern, B. S. Gossling, and R. H. Fowler, Proc. R. Soc. London, Ser. A 124, 699 (1929).

${ }^{9}$ G. L. R. Mair, J. Phys. D 17, 2323 (1984).

${ }^{10}$ D. R. Kingham and L. W. Swanson, Appl. Phys. A 34, 123 (1984).

${ }^{11}$ R. G. Forbes, Appl. Surf. Sci. 67, 9 (1993). 